01

\title{
Химический анализ зоопланктона методом лазерно-искровой эмиссионной спектроскопии без использования образцов сравнения
}

\author{
() Н.И. Сушков ${ }^{1}$, Н.В. Лобус ${ }^{2}$, И.В. Селиверстова ${ }^{1}$, Т.А. Лабутин ${ }^{1, \text { ศ }}$ \\ ${ }^{1}$ Московский государственный университет им. М.В. Ломоносова, химический факультет, \\ 119234 Москва, Россия \\ ${ }^{2}$ Институт океанологии им. П.П. Ширшова РАН, \\ 117997 Москва, Россия \\ ฯ e-mail: timurla@laser.chem.msu.ru
}

Поступила в редакцию 20.10.2019 г.

В окончательной редакции 07.02.2020 г.

Принята к публикации 29.05.2020 г.

Соотношения легких металлов ( $\mathrm{Li}, \mathrm{Na}, \mathrm{K}, \mathrm{Mg}$ и $\mathrm{Ca}$ ) в зоопланктоне (Calanus spp.) определены методом лазерно-искровой эмиссионной спектроскопии без использования образцов сравнения в предположении наличия локального термодинамического равновесия. Температуру плазмы, образующейся при лазерной абляции зоопланктона, оценивали по колебательно-вращательным полосам $\mathrm{CN}$, а электронную плотность по величине штарковского уширения линий $\mathrm{Mg}$ I $383.23 \mathrm{~nm}$, Li I $610.37 \mathrm{~nm}$ и Ca II $396.85 \mathrm{~nm}$. Модельные спектры лазерно-индуцированной плазмы в найденных условиях с учетом транспорта излучения позволили выбрать аналитические эмиссионные линии элементов, не подверженные самопоглощению. Полученные результаты сопоставлены с данными элементного анализа методами атомно-эмиссионной и масс-спектрометрии с индуктивно-связанной плазмой. Рассмотрено влияние равновесия ионизации на правильность результатов. Описанный способ предложено применять для прямого полуколичественного определения соотношений $\mathrm{Li}, \mathrm{Mg}$ и Са в зоопланктоне.

Ключевые слова: лазерно-искровая эмиссионная спектроскопия, химический анализ без образцов сравнения, диагностика плазмы, легкие металлы, ионизация, зоопланктон.

DOI: $10.21883 /$ OS.2020.09.49857.294-20

\section{Введение}

Анализ биологических образцов затруднен в силу высокой вариабельности состава и неоднородности матрицы. Кроме того, представляет интерес экспрессный анализ, не требующий значительных затрат времени и ресурсов. Чаще всего в биологических образцах методами атомной спектроскопии определяют тяжелые металлы, что необходимо для нужд экологического мониторинга [1]. Однако существует необходимость определения и легких элементов. В частности, отношение содержаний $\mathrm{Mg}$ и Са служит индикатором условий роста морских организмов [2]. Кроме того, в последние годы было установлено, что для арктических видов копепод рода Calanus характерно высокое накопление лития (наряду с мышьяком и ураном) [3,4]. При этом необходимо отметить, что соотношение легких металлов $\mathrm{Li} / \mathrm{Na}$ служит индикатором физиологического состояния организма, находящегося в состоянии диапаузы [5].

При прямом определении легких элементов в организмах планктона заметные преимущества по сравнению с другими методами имеет лазерно-искровая эмиссионная спектроскопия (ЛИЭС). Этот экспрессный метод не вызывает значительного разрушения образца, не требует сложной пробоподготовки, характеризуется хорошей чувствительностью к легким элементам (в отличие от рентгенофлуоресцентной спектроскопии, позволяющей, как правило, надежно определять только элементы тяжелее $\mathrm{Na}[6,7])$.

Уже имеется ряд работ, посвященных анализу методом ЛИЭС биологических образцов различной природы: растительных и животных тканей $[8,9]$, культур бактерий [10], пищевых продуктов [11]. Существенным ограничением для количественного анализа методом ЛИЭС является недостаток или совершенное отсутствие подходящих стандартных образцов состава (CO) для построения градуировочной зависимости. В частности, содержание лития в настоящий момент аттестовано всего в одном СО биологического материала - гомогенизате рыбы IAEA-407 (МАГАТЭ) [12].

В такой ситуации ЛИЭС позволяет рассчитать состав образца по интенсивностям эмиссионных линий без использования образцов сравнения (так называемый „безэталонный анализ“) [13]. В этом случае делается несколько важных допущений: соответствие состава плазмы испаряемой пробе, наличие локального термодинамического равновесия, оптически тонкая плазма. Поэтому для расчета состава образца требуется диагностика плазмы и тщательный выбор аналитических спектральных линий, свободных от самопоглощения. Помимо экспериментальной проверки самопоглощения линий, используется простой способ оценки наличия самопоглощения по отношению интенсивностей линий в мультиплете. Кроме того, предложено использовать расчетные коэффициенты поглощения для различных 
Таблица 1. Содержания элементов и золы в исследуемом образце зоопланктона (Calanus spp.)

\begin{tabular}{c|c}
\hline Элемент & Концентрация в $\mathrm{mg} / \mathrm{g}$ \\
\hline $\mathrm{Li}$ & 0.48 \\
$\mathrm{Na}$ & 43.65 \\
$\mathrm{Mg}$ & 4.49 \\
$\mathrm{~K}$ & 13.67 \\
$\mathrm{Ca}$ & 2.70 \\
$\mathrm{Sr}$ & 0.15 \\
$\mathrm{C}^{*}$ & $36 \%$ \\
Зола & $15.4 \%$
\end{tabular}

Примечание. * Органический углерод.

эмиссионных линий в лазерно-индуцированной плазме и выбирать линии, не подверженные самопоглощению в условиях конкретного эксперимента $[14,15]$.

В настоящей работе мы приводим первые результаты разработки способа определения легких металлов $(\mathrm{Li}$, $\mathrm{Na}, \mathrm{K}, \mathrm{Mg}$ и $\mathrm{Ca}$ ) в зоопланктоне методом ЛИЭС без использования образцов сравнения, а также обсуждаем источники погрешностей такого определения.

\section{Экспериментальная часть}

В работе использовались образцы, изготовленные из веслоногих рачков рода Calanus (преимущественно Calanus glacialis), отобранных в Восточно-Сибирском море. Элементный состав проб был определен методами атомно-эмиссионной и масс-спектрометрии с индуктивно-связанной плазмой [4]. Общий углерод определяли высокотемпературным сжиганием при температуре $900^{\circ} \mathrm{C}$ в токе воздуха, общий неорганический углерод - при $200^{\circ} \mathrm{C}$ с добавлением $\mathrm{H}_{3} \mathrm{PO}_{4}$ на анализаторе TOC 5000-V-CPH с использованием приставки SSM5000A (Shimadzu, Япония). Содержание органического углерода $\left(\mathrm{C}_{\mathrm{org}}\right)$ рассчитывали по разности общего и неорганического углерода [3-5]. Содержание золы определяли гравиметрическим методом после высокотемпературного сжигания органической матрицы образца $\left(550 \pm 25^{\circ} \mathrm{C}\right)$ в течение $6 \mathrm{~h}$ [4]. Результаты элементного анализа проб зоопланктона представлены в табл. 1. Для проведения измерений образцы рачков, высушенных при $50^{\circ} \mathrm{C}$ в течение $12 \mathrm{~h}$, прессовали в таблетки при небольшом давлении $(\sim 2 \mathrm{MPa})$.

Для экспериментов использовали установку, подробно описанную в работе [16]. Излучение 3-й гармоники $(\lambda=355 \mathrm{~nm}, 24 \mathrm{~mJ})$ Nd:YAG-лазера (LOTIS TII LS-134UTF, Беларусь) направляли на образец с использованием диэлектрических зеркал и кварцевых призм. Пучок фокусировали ахроматическим объективом $(f=151.5 \mathrm{~mm})$ на $6 \mathrm{~mm}$ ниже поверхности образца для повышения воспроизводимости в соответствии с рекомендациями, изложенными в работе [17]. Излучение плазмы проецировали двухлинзовым конденсором на входную щель $(25 \mu \mathrm{m})$ спектрографа Черни-
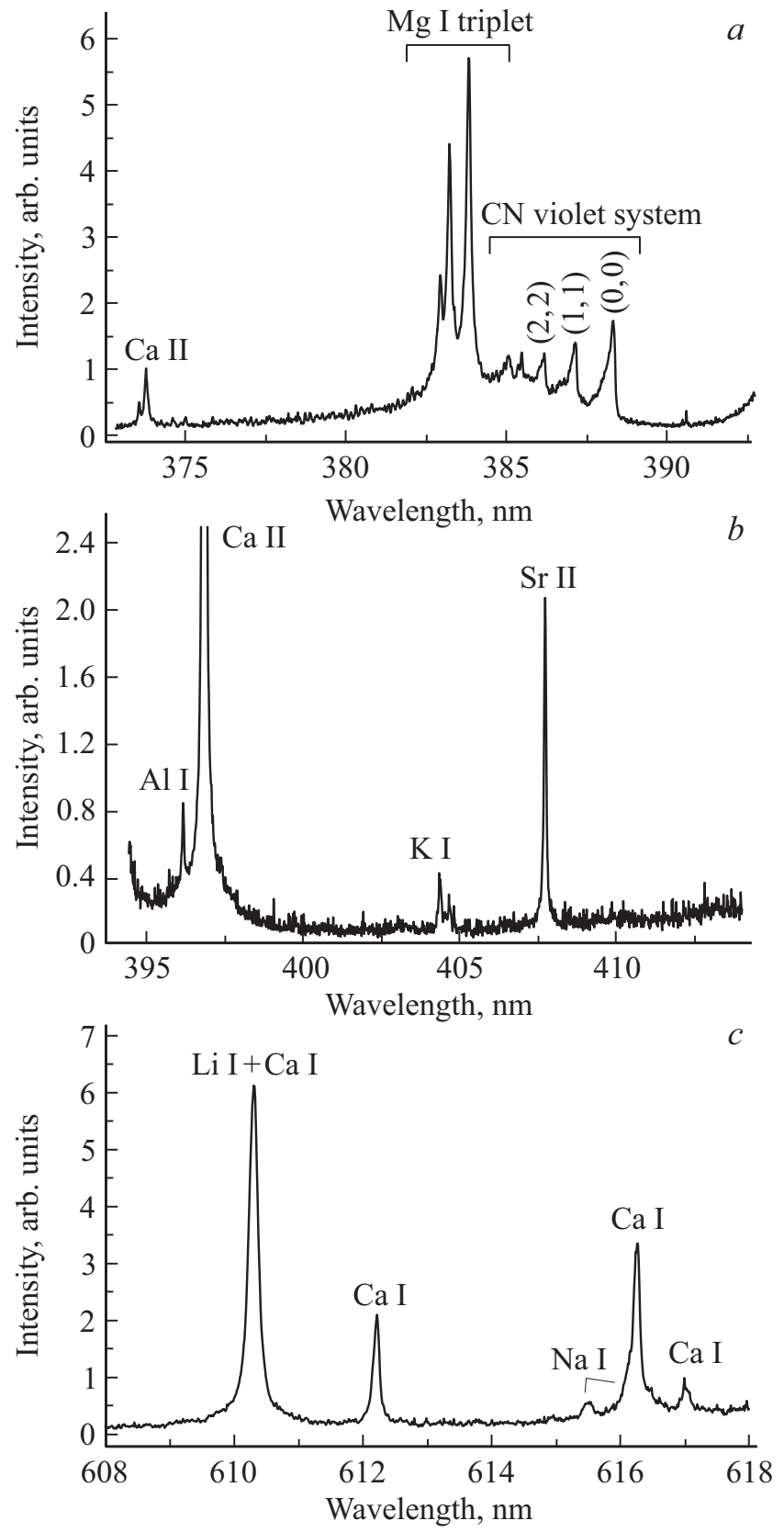

Рис. 1. Фрагменты спектра плазмы зоопланктона в диапазонах $373-393 \mathrm{~nm}(a), 394-415 \mathrm{~nm}(b)$ и $608-618 \mathrm{~nm}(c)$.

Тернера HR 320 (ISA Instruments, США, $f=320 \mathrm{~mm}$, $1800 \mathrm{gr} / \mathrm{mm}, R=10800$ на длине волны $589 \mathrm{~nm})$. Спектры (рис. 1) регистрировали с помощью цифровой стробируемой камеры „Наногейт-2В“ (НПО „Наноскан“, Россия) с усилителем яркости, управляемой разработанным в лаборатории программным обеспечением.

\section{Обсуждение результатов}

Чтобы выбрать условия для аналитических измерений, мы изучили временную эволюцию лазерноиндуцированной плазмы, образующейся при абляции 

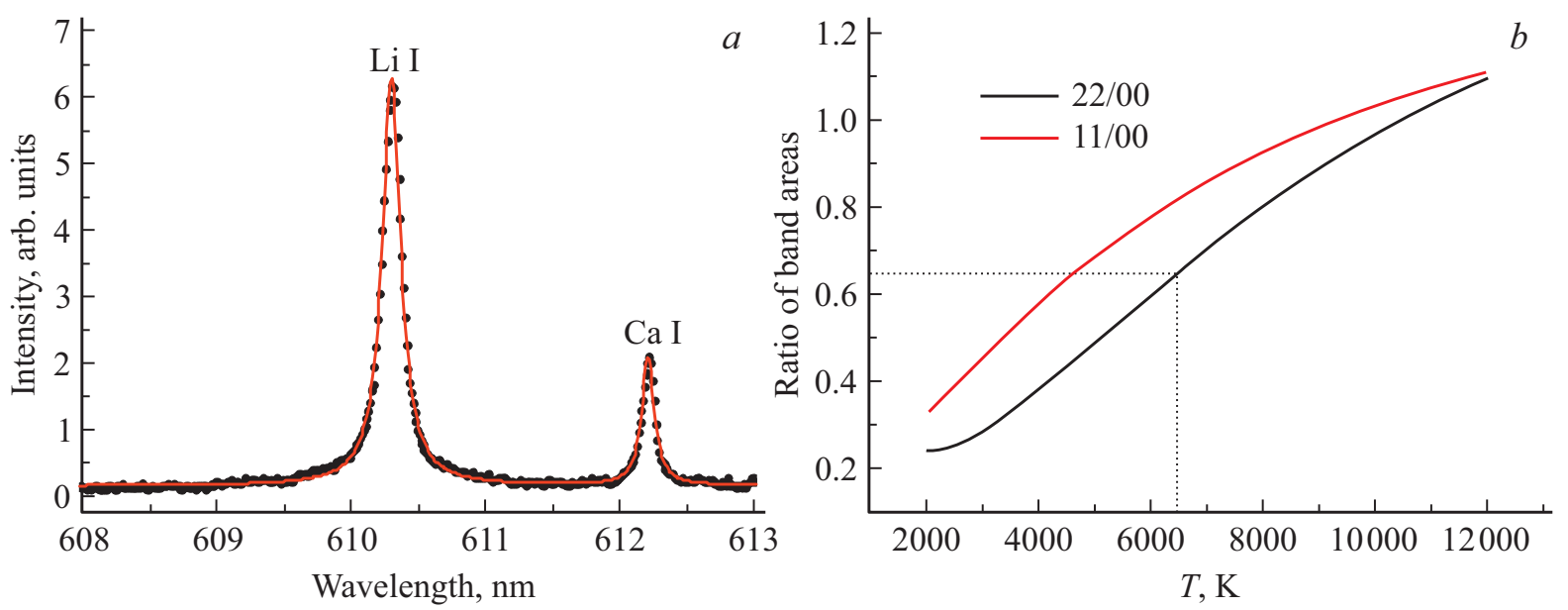

Рис. 2. $a-$ аппроксимация контуром Лоренца (красная линия) экспериментальных профилей линий Li I и Ca I при 610-612 nm (черные точки); $b$ - теоретически рассчитанные номограммы зависимостей соотношений площадей ЭКВ полос CN от температуры.

Таблица 2. Ударные штарковские параметры для линий металлов (при $5000 \mathrm{~K}$ ) при $N_{e}=10^{16} \mathrm{~cm}^{-3}$ [19]

\begin{tabular}{c|c}
\hline Линия, $\mathrm{nm}$ & $w_{e}, \AA$ \\
\hline Li I 610.37 & 0.213 \\
Mg I 383.23 & 0.107 \\
Ca II 396.85 & 0.190
\end{tabular}

рачков, в интервале $0.5-3 \mu \mathrm{s}$. Электронную плотность оценивали по штарковскому уширению линий со значительным ударным штарковским параметром (табл. 2): Mg I $383.23 \mathrm{~nm}$, Li I $610.37 \mathrm{~nm}$ и Сa II $396.85 \mathrm{~nm}$. Профили линий аппроксимировали контуром Лоренца (рис. 2,a), допплеровским уширением пренебрегали (при максимальной экспериментальной температуре оно не превышало 17 pm, что значительно меньше ширины инструментальной функции). Из полученной величины вычитали инструментальную ширину на данной длине волны [18]. В случае линий магния, кальция и лития пренебрегали ионным уширением и дебаевским экранированием, т.е. считали, что их штарковская ширина прямо пропорциональна электронной плотности [19]:

$$
F W H M_{S}\left(N_{e}, T\right)=2 w_{e}(T) 10^{-16} N_{e}
$$

где $w_{e}$ - электронный ударный параметр [20].

Для расчета температуры плазмы мы воспользовались молекулярными эмиссионными полосами $\mathrm{CN}$ (фиолетовая система $B^{2} \Sigma^{+}-X^{2} \Sigma^{+}$с $\Delta v=0$ при $372-389 \mathrm{~nm}$, рис. $1, a)$, предполагая существование локального термодинамического равновесия (равенство электронной, колебательной и вращательной температур). В этом случае, рассматривая относительные интенсивности переходов внутри данной электронно-колебательно-враща- тельной (ЭКВ) полосы, мы можем записать [21]:

$$
I_{i}=C \sum_{\substack{v^{\prime}, J^{\prime} \\ v^{\prime \prime}, J^{\prime \prime}}} \tilde{v}^{4} \phi \exp \left[-\frac{h c F\left(n^{\prime}, v^{\prime}, J^{\prime}\right)}{k_{B} T}\right] f\left(\tilde{v}, \tilde{v}_{i}\right)
$$

Здесь $I_{i}-$ интенсивность сигнала на $i$-м элементе детектора, $C$ - константа, $\tilde{v}-$ волновое число перехода $\left(\mathrm{cm}^{-1}\right), \tilde{v}_{i}-$ волновое число, соответствующее данному элементу детектора, $\phi-$ произведение факторов Франка-Кондона и Хенля-Лондона для данного перехода, $F$ - энергия ЭКВ терма $\left(\mathrm{cm}^{-1}\right), n^{\prime}, v^{\prime}, J^{\prime}-$ соответственно электронное, колебательное и вращательное квантовое число начального состояния перехода, $v^{\prime \prime}, J^{\prime \prime}$ - квантовые числа конечного состояния перехода, $k_{B}$ - постоянная Больцмана, $f\left(\tilde{v}, \tilde{v}_{i}\right)-$ контур аппаратной функции (для нашего оборудования этот контур наилучшим образом описывается функцией Лоренца с шириной $4.1 \mathrm{~cm}^{-1}$ в данном спектральном диапазоне). Энергия ЭКВ терма выражается следующим образом:

$$
\begin{aligned}
& F=T_{e}+\omega_{e}\left(v+\frac{1}{2}\right)-\omega_{e} x_{e}\left(v+\frac{1}{2}\right)^{2}+\ldots \\
& +B_{v} J(J+1)-D_{v} J^{2}(J+1)^{2}+H_{v} J^{3}(J+1)^{3}+\ldots,
\end{aligned}
$$

где $T_{e}-$ электронная энергия, $\omega_{e}$ и $\omega_{e} x_{e}-$ колебательные постоянные, $B_{v}, D_{v}$ и $H_{v}$ - вращательные постоянные [22]. Расчет теоретических эмиссионных ЭКВ спектров проводили в соответствии с рекомендациями [21] с помощью алгоритма, разработанного в среде GNU Octave. При этом учитывали все переходы с колебательными квантовыми числами $v^{\prime}$ от 0 до 9 и все вращательные состояния $J^{\prime}, J^{\prime \prime}$ [23-25] с энергией ниже диссоциационного предела основного электронного состояния $(7.5 \mathrm{eV})$. Первоначально температуру рассчитывали наиболее простым способом - по номограммам зависимостей соотношений интенсивности полос от 


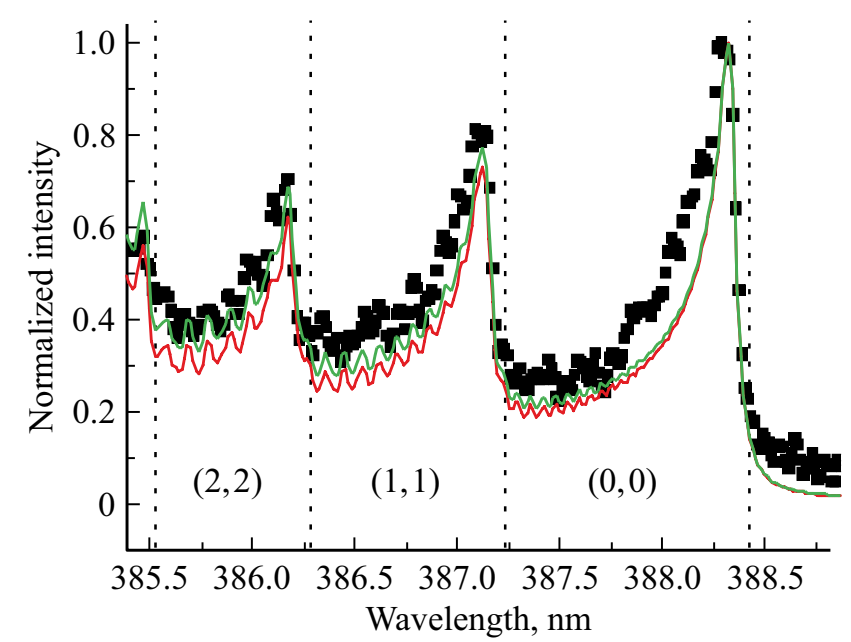

Рис. 3. Спектр плазмы зоопланктона при задержке регистрации $1.5 \mu \mathrm{s}$ : фрагмент фиолетовой системы $\mathrm{CN}\left(B^{2} \Sigma^{+}-X^{2} \Sigma^{+}\right.$, $\Delta v=0)$ - эксперимент (черные точки), модельный спектр при температуре, полученной из номограмм (красная линия), аппроксимации экспериментальных данных методом перебора (зеленая линия). Штриховыми линиями показаны положения кантов электронно-колебательно-вращательных полос.

Таблица 3. Сопоставление результатов элементного анализа зоопланктона методом ЛИЭС без образцов сравнения с данными ИСП-МС (1 - совпадение результатов $)$

\begin{tabular}{|c|c|c|c|c|c|}
\hline Задержка, $\mu \mathrm{s}$ & \multirow[t]{2}{*}{0.5} & \multirow[t]{2}{*}{0.75} & \multirow[t]{2}{*}{1.0} & \multirow[t]{2}{*}{1.25} & \multirow[t]{2}{*}{1.5} \\
\hline Пара линий & & & & & \\
\hline Na I 330.237/Li I 610.365 & 0.220 & 0.225 & 0.101 & 0.166 & 0.178 \\
\hline K I 404.413/Li I 610.365 & 0.730 & 0.431 & 0.730 & 0.792 & 0.519 \\
\hline Ca I 612.222/Li I 610.365 & 2.135 & 1.814 & 1.823 & 1.728 & 1.660 \\
\hline Mg I 383.230/Li I 610.365 & 0.874 & 1.096 & 0.393 & 1.824 & 0.661 \\
\hline K I $404.413 / \mathrm{Na}$ I 330.237 & 3.32 & 1.91 & 7.20 & 4.78 & 2.91 \\
\hline $\mathrm{Ca}$ I $612.222 / \mathrm{Mg}$ I 517.268 & 1.391 & 1.632 & 1.083 & 0.810 & 0.889 \\
\hline СКО & 0.46 & 0.28 & 1.06 & 0.67 & 0.38 \\
\hline
\end{tabular}

температуры. Номограммы предварительно рассчитали с шагом $25 \mathrm{~K}$ в интервале от 2000 до $12000 \mathrm{~K}$ для соотношений интенсивности полос $(1-1 / 0-0)$ и $(2-2 / 0-0)$ (рис. $2, b$ ). Пределы интегрирования площадей полос для расчета интенсивности составляли $387.23-388.42(0-0)$, $386.28-387.23$ (1-1) и $385.52-386.28 \mathrm{~nm}(2-2)$.

Также для определения температуры нами был использован простейший вариант аппроксимации экспериментального спектра методом перебора с равномерным шагом $(50 \mathrm{~K}$, диапазон $3000-12000 \mathrm{~K}$, рис. 3). Экспериментальный и модельные спектры нормировали на интервал $[0,1]$ по интенсивности, после чего вычисляли квадраты их разностей для каждого значения длины волны. Зависимость суммы этих квадратов от $T$ имеет глобальный минимум, что позволяет найти единственное решение и таким образом определить температуру лазерно-индуцированной плазмы.
Температуры, рассчитанные описанными двумя способами, были в целом близки друг к другу (рис. 4, $a$ ). Однако на поздних временах наблюдения (более $1.5 \mu \mathrm{s}$ ) температура, получаемая при аппроксимации спектров, остается практически неизменной, что не согласуется с охлаждением плазмы при расширении и теплообмене с окружающим газом. Возможно, это связано с мешающим влиянием нескольких относительно малоинтенсивных линий кальция, железа и других элементов, лежащих в этой области. Из-за использования более узкого спектрального диапазона метод номограмм оказался меньше подвержен влиянию помех, так что расчет колебательной температуры проводили с его использованием. Соответственно в дальнейшей части работы использовали среднее арифметическое, полученное из результатов определения $T$ по номограммам.

Величины электронной плотности, рассчитанные по штарковской ширине линий металлов, близки и монотонно снижаются по мере расширения и охлаждения лазерного факела (рис. 4,b). Для характеризации плазмы на временах $0.5-1.5 \mu$ s выбрали значения $N_{e}$, полученные из ширины линии $\mathrm{Mg}$ I $383.230 \mathrm{~nm}$, так как профиль линии Са II $396.847 \mathrm{~nm}$ может быть искажен из-за самопоглощения, а линия Li I $610.365 \mathrm{~nm}$ в некоторых случаях описывалась контуром Лоренца с большей погрешностью, чем Mg I.

Выбор линий осуществляли на основании модельных спектров плазмы, рассчитываемых по разработанному ранее в нашей лаборатории алгоритму, учитывающему поглощение излучения в плазме [15]. Были рассмотрены линии с низким уровнем самопоглощения и спектральных помех (пропускание не менее 0.9, относительный вклад спектральных наложений в наблюдаемую интенсивность не более 0.05). На рис. 1 изображены области спектра с выбранными линиями, которые были достаточно интенсивны в экспериментальных спектрах для проведения количественного анализа. Литий и кальций определяли по линиям при 610.37 и $612.22 \mathrm{~nm}$ соответственно. В сигнал лития вносили поправку на перекрывающуюся с ней линию Са I $610.272 \mathrm{~nm}$ с использованием интенсивности линии кальция Са I $612.22 \mathrm{~nm}$. Резонансная линия Li I $670.783 \mathrm{~nm}$ не могла быть использована из-за значительного самопоглощения. В случае калия использовали коротковолновую компоненту дублета (при $404.414 \mathrm{~nm}$ ). Кроме показанных на рис. 3 линий, в расчете участвовали также линии $\mathrm{Na}$ I при $330.237 \mathrm{~nm}$ и $\mathrm{Mg}$ I при 383.230 и $517.268 \mathrm{~nm}$.

Мы определяли соотношения элемент/литий для элементов $\mathrm{Na}, \mathrm{K}, \mathrm{Ca}$ и $\mathrm{Mg}$, а также соотношения $\mathrm{K} / \mathrm{Na}$ и $\mathrm{Ca} / \mathrm{Mg}$. При расчетах предполагали стехиометрическое испарение образца, равновесное заселение электронных уровней, а также учитывали ионизацию элементов до второй ступени включительно: в соответствии с уравнением Саха [26] рассчитывали отношения объемных концентраций однозарядных ионов и нейтральных атомов $\alpha_{1}=n_{\text {II }} / n_{\text {I }}$, двухзарядных и однозарядных ионов $\alpha_{2}=n_{\text {III }} / n_{\text {II }}$, а суммарную степень ионизации рассчиты- 
Таблица 4. Доли неионизованных атомов $(1-\alpha)$ и их соотношения $(1-\alpha)_{i} /(1-\alpha)_{j}$ для задержки $0.75 \mu \mathrm{s}$

\begin{tabular}{c|ccccc|cccccc}
\hline$T, \mathrm{~K}$ & $\mathrm{Li}$ & $\mathrm{Na}$ & $\mathrm{K}$ & $\mathrm{Mg}$ & $\mathrm{Ca}$ & $\mathrm{Na} / \mathrm{Li}$ & $\mathrm{K} / \mathrm{Li}$ & $\mathrm{Mg} / \mathrm{Li}$ & $\mathrm{Ca} / \mathrm{Li}$ & $\mathrm{K} / \mathrm{Na}$ & $\mathrm{Ca} / \mathrm{Mg}$ \\
\hline 8500 & 8.3 & 7.3 & 4.1 & 30 & 7.8 & 0.88 & 0.49 & 3.61 & 0.93 & 0.56 & 0.26 \\
7800 & 15 & 12 & 5.9 & 53 & 14 & 0.80 & 0.40 & 3.53 & 0.95 & 0.49 & 0.27 \\
7140 & 29 & 22 & 9.6 & 78 & 28 & 0.77 & 0.34 & 2.73 & 0.97 & 0.44 & 0.36
\end{tabular}
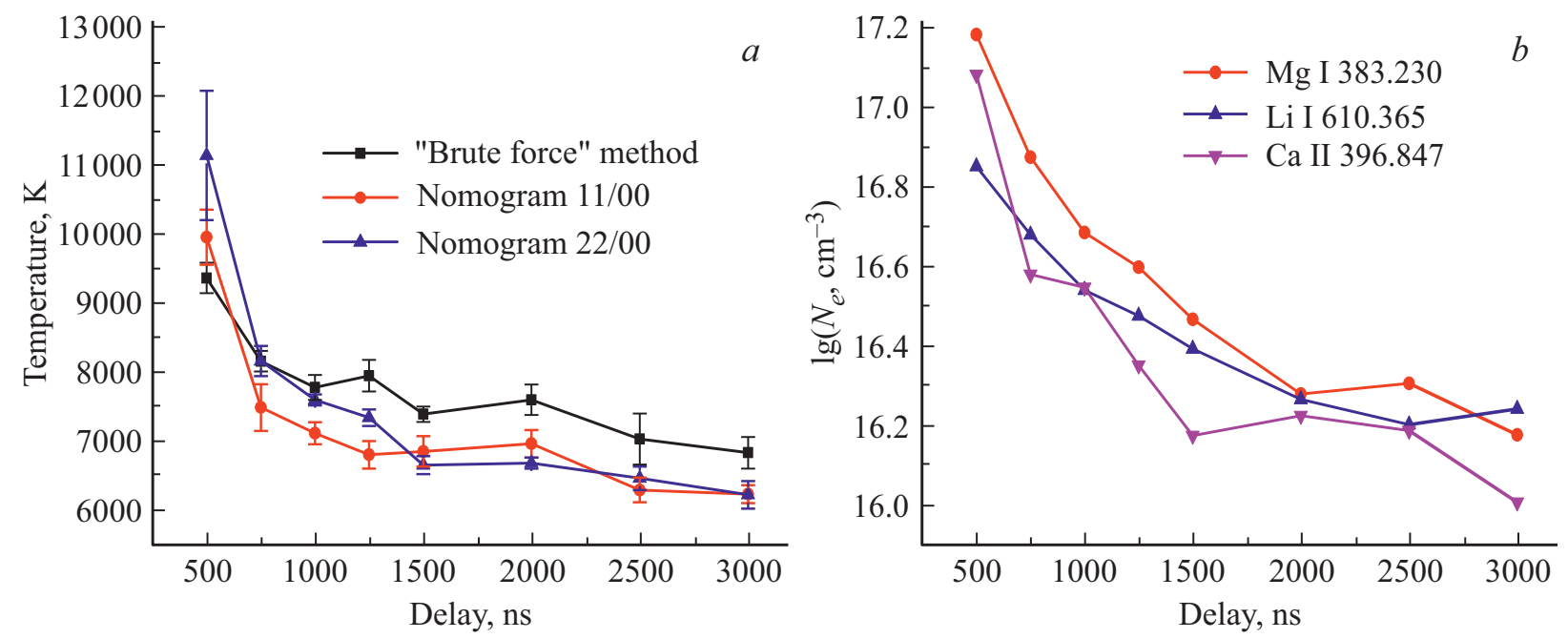

Рис. 4. $a$ - эволюция температуры плазмы зоопланктона во времени (расчет по соотношению площадей полос $(1,1)$ и $(0,0)-$ красная линия; $(2,2)$ и $(0,0)$ - синяя линия; аппроксимация экспериментального спектра методом перебора - черная линия). $b-$ картина временно́й эволюции электронной плотности в плазме зоопланктона, полученная по разным спектральным линиям.

вали как

$$
\begin{aligned}
\alpha & =\left(n_{\mathrm{III}}+n_{\mathrm{II}}\right) /\left(n_{\mathrm{I}}+n_{\mathrm{II}}+n_{\mathrm{III}}\right)= \\
& =\alpha_{1}\left(1+\alpha_{2}\right) /\left(1+\alpha_{1}\left(1+\alpha_{2}\right)\right) .
\end{aligned}
$$

При таких допущениях расчет массовых соотношений элементов можно проводить по следующей формуле $[13,14]$ :

$$
\frac{c_{\mathrm{Li}}}{c_{\mathrm{Na}}}=\frac{I_{\lambda(\mathrm{LiI})} Z_{\mathrm{Li}}(g A)_{\mathrm{Na}} \exp \left(-\frac{E_{\mathrm{Na}}}{k T}\right) \alpha_{\mathrm{Na}} \lambda(\mathrm{Li} \mathrm{I})}{I_{\lambda(\mathrm{NaI})} Z_{\mathrm{Na}}(g A)_{\mathrm{Li}} \exp \left(-\frac{E_{\mathrm{Li}}}{k T}\right) \alpha_{\mathrm{LiI}} \lambda(\mathrm{NaI})},
$$

где $I$ - интенсивность, $Z$ - статистическая сумма, $g$ статистический вес уровня, $A-$ вероятность перехода, $\alpha$ - степень ионизации $[13,26]$. В табл. 3 приведены отношения полученных результатов к значениям, полученным независимым методом (ИСП-МС). Таким образом, чем ближе полученное число к 1 , тем лучше правильность определения.

Согласно табл. 3, лучшие результаты (минимум в графе СКО) получаются при задержке $0.75 \mu \mathrm{s}$. Это можно объяснить, в частности, высоким отношением сигнала к шуму при относительно высоких температурах плазмы и высокой электронной плотностью, обеспечивающей состояние равновесного заселения электронных уровней. Расхождения могут быть обусловлены недостаточной точностью определения параметров плазмы, которые необходимы для правильной оценки заселенности энергетических уровней и степени ионизации элементов. При наблюдаемой температуре $7800 \mathrm{~K}$ и $N_{e}=7.5 \cdot 10^{16} \mathrm{~cm}^{-3}$ степень ионизации $(\alpha)$ рассматриваемых элементов составляет около $90 \%$, кроме $\mathrm{Mg}$ (48\%). Поскольку получить аналитическое выражение для расчета погрешности практически невозможно изза сложности дифференцирования сумм по состояниям, можно рассмотреть размах значений степени ионизации, обусловленный погрешностью температуры (табл. 4). Соответственно для каждой экспериментальной точки были рассчитаны значения $\alpha$ при трех температурах: среднем значении $T$ и на границах доверительного интервала $(p=0.95)$. Так, при изменении температуры с 7100 до $8500 \mathrm{~K} \alpha$ изменяется от 70 до $92 \%$ в случае $\mathrm{Li}$ и от 20 до $70 \%$ для $\mathrm{Mg}$. Таким образом, в условиях нашего эксперимента доля незаряженных атомов $(1-\alpha)$, излучающих на выбранных нами аналитических длинах волн, оказывается небольшой и заметно зависимой прежде всего от температуры плазмы, что хорошо согласуется с данными моделирования спектров плазмы [15]. Таким образом, погрешность оценки температуры вносит большую неопределенность в результат расчета состава образца. Расчеты для всех задержек в интервале $0.5-1.5 \mu$ с смотри в дополнительных материалах (табл. ESM_1.xlsx).

Можно заметить, что для соотношений элементов влияние степени ионизации существенно меньше, в частности, отношение $\mathrm{Li} / \mathrm{Ca}$ является самым устойчивым во времени из всех рассчитанных (размах менее 5\%). 
Несмотря на выбор линий, ограничивающий влияние самопоглощения, этот эффект все равно мог сказаться на характеристиках правильности, так как реальная плазма устроена сложнее, чем предполагается при моделировании (в частности, она не является однородной, изотропной и сферической). Возможны и ошибки при определении некоторых элементов (например, $\mathrm{Na}$ ) независимым методом. Можно считать, что рассмотрение ионизационного равновесия удовлетворительно объясняет экспериментальные данные. Таким образом, для проведения аналитических измерений необходимо выбирать временные интервалы наблюдения плазмы, в которых вариабельность степени ионизации меньше.

Для более низких температур $\alpha$ станет достаточно малой $(<10 \%)$ только при $T<4000 \mathrm{~K}$. Такие условия реализуются при достаточно больших задержках (порядка $8-10 \mu \mathrm{s})$. Однако в этих условиях, во-первых, отношение сигнала к шуму будет слишком мало для достоверной регистрации сигнала некоторых элементов, а во-вторых, уже не будет ЛТР, необходимым условием которого является выполнение критерия Мак-Виртера для $N_{e}[27]$ :

$$
N_{e}>\frac{2.55 \cdot 10^{11}}{\langle\bar{g}\rangle} T^{1 / 2}(\Delta E)^{3} .
$$

Здесь $T$ выражена в $\mathrm{K},\langle\bar{g}\rangle$ - безразмерный фактор Ганта, $\Delta E-$ разность энергий начального и конечного уровней перехода. В частности, для линии $\mathrm{Na}$ I при $330 \mathrm{~nm}$ значение критерия при $4000 \mathrm{~K}$ равно $3.6 \cdot 10^{16} \mathrm{~cm}^{-3}$, тогда как уже через $2.5 \mu \mathrm{s}$ после лазерного импульса электронная плотность составляет всего $1.6 \cdot 10^{16} \mathrm{~cm}^{-3}$.

Для снижения погрешности, вносимой электронной плотностью, ее было бы предпочтительно определять по линии Н-альфа, которая имеет наибольший параметр штарковского уширения и тем самым обеспечивает наименьшую погрешность, однако неоднородность плазмы мешает воспользоваться этой линией при определении металлов.

На настоящий момент методика позволяет проводить полуколичественное определение упомянутых соотношений. Исходя из вышесказанного, очень важной задачей представляется снижение погрешностей при диагностике плазмы путем получения как можно большего количества спектров (для повышения отношения сигнала к шуму) и их тщательной статистической обработки (с целью удаления выбросов). То же можно сказать и о регистрации собственно аналитических линий элементов, тем более что поверхность изучаемых образцов существенно неоднородна и по составу, и по физическим свойствам. Такое требование подразумевает довольно большой расход образца, который из-за своих механических свойств сильно повреждается лазерными импульсами (глубокие кратеры образуются уже после 1-2 импульсов в одной точке, частицы образца разбрасываются ударной волной, что ведет к изменению условий пробоотбора). Другой путь решения проблемы - работа в условиях более гомогенной плазмы, реализующихся на более поздних временах ее жизни (в нашем случае более $2 \mu \mathrm{s}$ ). Однако в этом случае падает общая интенсивность сигнала и могут нарушаться условия ЛТР. Таким образом, выбор условий эксперимента требует поиска определенного компромисса между перечисленными факторами.

\section{Выводы}

Предпринятая попытка провести анализ зоопланктона методом ЛИЭС без использования образцов сравнения показала, что для данного типа образцов этот подход позволяет проводить полуколичественное определение отношений элементов на ранних временах существования лазерного факела (до $1 \mu \mathrm{s}$ ), однако химический состав и физические свойства образца затрудняют диагностику температуры и электронной плотности с высокой точностью и снижают воспроизводимость из-за быстрого формирования глубокого кратера. Возможно, использование более коротковолнового излучения (например, 4-й гармоники неодимового лазера с $\lambda=266 \mathrm{~nm}$ ) позволит в дальнейшем добиться более благоприятных условий формирования аналитического сигнала за счет более эффективной атомизации пробы (коэффициент поглощения излучения образцом обратно пропорционален длине волны [28]). Погрешности определения температуры приводят к большой неопределенности расчетных степеней ионизации элементов и таким образом ухудшают характеристики правильности анализа. Для большинства изученных соотношений элементов эта неопределенность минимальна при температурах около $7400 \mathrm{~K}$ $\left(N_{e} \sim 3 \cdot 10^{16} \mathrm{~cm}^{-3}\right)$. Кроме того, некоторые соотношения (например, $\mathrm{Ca} / \mathrm{Li}$ ) обнаруживают устойчивость к изменению условий в широком диапазоне, что связано с индивидуальными особенностями структуры электронных уровней энергии и благоприятными соотношениями потенциалов ионизации соответствующих элементов.

Возможные пути решения указанных проблем выбор временных параметров регистрации сигнала с учётом степени ионизации определяемых компонентов, использование большего числа импульсов для регистрации спектров (чтобы повысить отношение сигнала к шуму после усреднения данных), тщательная статистическая обработка спектров (в первую очередь, удаление выбросов), а также использование для абляции коротковолнового лазерного излучения, что позволит улучшить морфологию кратера и степень атомизации аблированного вещества в лазерно-индуцированной плазме.

\section{Финансирование работы}

Исследование выполнено при финансовой поддержке Российского фонда фундаментальных исследований в рамках научного проекта № 18-33-01297. Анализы 
зоопланктона методами ИСП-АЭС, ИСП-МС, органического углерода и золы выполнены за счет средств Российского научного фонда (проект № 18-77-00064).

\section{Конфликт интересов}

Авторы заявляют, что у них нет конфликта интересов.

\section{Список литературы}

[1] Зоров Н.Б., Попов А.М., Зайщев С.М., Лабутин Т.А. // Усп. химии. 2015. Т. 84. № 6. С. 1021; Zorov N.B., Popov A.M., Zaytsev S.M., Labutin T.A. // Russ. Chem. Rev. 2015. V. 84. N 6. P. 1021.

[2] Marali S. et al. // Palaeogeogr. Palaeoclimatol. Palaeoecol. 2017. V. 484. P. 109.

[3] Лобус Н.В. // Океанология. 2016. Т. 56. № 6. С. 890; Lobus N.V. // Oceanology. 2016. V. 56. N 6. P. 809.

[4] Lobus N.V., Arashkevich E.G., Flerova E.A. // Environmental Science and Pollution Research. 2019. V. 26. Iss. 22. P. 23044.

[5] Лобус Н.В., Дрии, А.В., Флинт М.В. // Океанология. 2018. T. 58. № 3. C. 318; Lobus N.V., Drits A.V., Flint M.V. // Oceanology. 2018. V. 58. N 3. P. 405.

[6] Penner-Hahn J.E. Handbook of X-ray Spectrometry. NY.; Basel: Marcel Dekker, Inc., 2002. 984 p.

[7] Павлинский Г.В. Основы физики рентгеновского излучения. М.: Физматлит, 2007. 240 с.

[8] Santos D. et al. // Spectrochim. Acta. Part B. 2012. V. 71-72. P. 3.

[9] Kaiser J. et al. // Surf. Sci. Rep. 2012. V. 67. N 11-12. P. 233.

[10] Prochazka D. et al. // Spectrochim. Acta. Part B. 2018. V. 139. P. 6.

[11] Markiewicz-Keszycka M. et al. // Trends Food Sci. Technol. 2017. V. 65. P. 80.

[12] IAEA-407, Fish Homogenate (Methyl Mercury) [электронный ресурс]. Режим доступа: https://nucleus.iaea.org/rpst/ ReferenceProducts/ReferenceMaterials/ Trace_Elements_Methylmercury/IAEA-407.htm

[13] Ciucci A. et al. // Appl. Spectrosc. 1999. V. 53. N 8. P. 960.

[14] Tognoni E., Cristoforetti G., Legnaioli S., Palleschi V. // Spectrochim. Acta. Part B. 2010. V. 65. N 1. P. 1.

[15] Zaytsev S.M., Popov A.M., Labutin T.A. // Spectrochim. Acta. Part B. 2019. V. 158. N 105632.

[16] Zaytsev S.M., Popov A.M., Zorov N.B., Labutin T.A. // J. Instrum. 2014. V. 9. N 06. P. 06010.

[17] Aragón C., Aguilera J.A., Peñalba F. // Appl. Spectrosc. 1999. V. 53. N 10. P. 1259.

[18] Aragón C., Aguilera J.A. // Spectrochim. Acta. Part B. 2008. V. 63. N 9. P. 893.

[19] Konjević N. // Phys. Rep. 1999. V. 316. N 6. P. 339.

[20] Griem H.R. Spectral Line Broadening by Plasmas. NY.; London: Academic Press, 1974. 410 p.

[21] Hornkohl J.O., Parigger C., Lewis J.W.L. // J. Quant. Spectrosc. Radiat. Transf. 1991. V. 46. N 5. P. 405.

[22] Huber K.P., Herzberg G. Molecular Spectra and Molecular Structure. V. IV. Constants of diatomic molecules. Ottawa: Van Nostrand Reinhold Company, 1979. 716 р.; Хьюбер K.П., Герцберг Г. Константы двухатомных молекул. В 2-х ч. Ч. 1. М.: Мир, 1984. 408 с.

[23] Очкин В.Н. Спектроскопия низкотемпературной плазмы. М.: Физматлит, 2006. 472 с.
[24] Ito H. et al. // J. Mol. Spectrosc. 1988. V. 127. N 2. P. 283.

[25] Kotlar A.J., Field R.W., Steinfeld J.I. // J. Mol. Spectrosc. 1980. V. 80. N 1. P. 86.

[26] Hill S.J. (ed.). Inductively Coupled Plasma Spectrometry and its Applications. Blackwell Publishing, 2007. 448 p.

[27] Cristoforetti G. et al. // Spectrochim. Acta. Part B. 2010. V. 65. P. 86.

[28] von Allmen M., Blatter A. Laser-beam interactions with materials. Physical principles and applications. Springer, 1998. $194 \mathrm{p}$. 